\title{
Synergistic inhibitory activity of zoledronate and paclitaxel on bone metastasis in nude mice
}

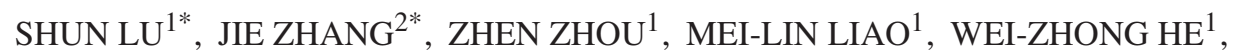 \\ XIAO-YAN ZHOU ${ }^{2}$, ZI-MING LI ${ }^{1}$, JIA-QING XIANG ${ }^{2}$, JIE-JUN WANG ${ }^{3}$ and HAI-QUAN CHEN ${ }^{2}$ \\ ${ }^{1}$ Shanghai Lung Cancer Center, Shanghai Chest Hospital, Jiao Tong University, Shanghai 200030; ${ }^{2}$ Department of \\ Thoracic Surgery, Shanghai Cancer Hospital, Fudan University, Department of Oncology, Shanghai Medical College, \\ Fudan University, Shanghai 200032; ${ }^{3}$ Department of Medical Oncology, Changzheng Hospital, \\ Second Military Medical University, Shanghai 200070, P.R. China
}

Received January 24, 2008; Accepted April 3, 2008

DOI: 10.3892/or_00000045

\begin{abstract}
Zoledronic acid (Zometa, ZOL) and cytotoxic chemotherapy agents have been reported to have synergistic antitumor activities. However, there is limited data on the effects of combination therapies on the development of bone metastasis in animal models of lung cancer. The purpose of this study was to establish a human lung adenocarcinoma cell line with high bone metastatic potential in an immunodeficient mouse model and to evaluate the synergistic inhibitory activity of zoledronate and paclitaxel $(\mathrm{P})$ on bone metastasis in nude mice. A human lung adenocarcinoma cell line with high bone metastatic potential (SPC-A1-BM) was established by 10 rounds of in vivo selection. Cells were inoculated into the cardiac ventricle of NIH-BNX mice, which were treated 8 days later with: ZOL $(0.2 \mathrm{mg} / \mathrm{kg}$ s.c. twice weekly) alone, $\mathrm{P}$ (6.0 mg/kg every week, i.p.) alone, $\mathrm{P}$ + ZOL, or vehicle (10 mice per group). Tumor growth was evaluated with bone scans, $\mathrm{X}$-rays and in situ immunohistochemistry. Serum n-telopeptide of type I collagen (NTX) was measured by ELISA. Survival was assessed using the Kaplan-Meier method. Bone scan, radiographic and histological assessments revealed fewer bone metastases in all treatment groups vs. vehicle, with $\mathrm{P}+\mathrm{ZOL}$ significantly reducing the incidence of bone metastases detected by bone scans $(P=0.020)$ and $X$-rays $(P=0.036)$. A histological analysis revealed marginal differences in the number of bone metastases between $\mathrm{P}+\mathrm{ZOL}$ and vehicle $(\mathrm{P}=0.058)$. There
\end{abstract}

Correspondence to: Professor Haiquan Chen, Department of Thoracic Surgery, Shanghai Cancer Hospital, Fudan University, Shanghai 200030, P.R. China

E-mail: zhangjie2289@ hotmail.com

Professor Jie-jun Wang, Department of Medical Oncology, Changzheng Hospital, Second Military Medical University, Shanghai 200070, P.R. China

E-mail: zhangjie2289@hotmail.com

${ }^{*}$ Contributed equally

Key words: non-small cell lung cancer, bone metastasis, zoledronate was a trend towards differences in survival between the groups $(\mathrm{P}=0.1511)$ and survival was significantly longer for the $\mathrm{P}+\mathrm{ZOL}$ group vs. vehicle $(\mathrm{P}=0.022)$. Compared with vehicle and $\mathrm{ZOL}$ alone, cancerous cells in the bone of mice treated with $\mathrm{P}+\mathrm{ZOL}$ expressed higher levels of Bax and lower levels of Bcl-2 and Bcl-xl. ZOL produced a trend towards reduced NTX levels vs. vehicle and P + ZOL produced a profound reduction in NTX vs. vehicle $(\mathrm{P}=0.022)$. The results of this study indicated that zoledronate enhanced the efficacy of paclitaxel synergistically, by reducing the incidence of bone metastasis from lung cancer and prolonging survival in a mouse model of non-small cell lung cancer with a high potential for metastasis to bone.

\section{Introduction}

Bone seems a milieu particularly favorable for the growth, vascularization and survival of certain kinds of cancer $(1,2)$. Approximately $30-65 \%$ of advanced non-small cell lung cancer (NSCLC) patients have bone metastasis and the consequences are often devastating $(3,4)$. It can cause severe pain, pathological fractures, life-threatening hypercalcemia, spinal cord compression and other nerve-compression syndromes (5).

Based on many clinical trials, intravenous bisphosphonate therapy proves to be safe for long-term use and provides durable treatment benefits. The second- and third-generation nitrogen-containing bisphosphonate inhibits protein prenylation in osteoclasts (6). One such third-generation bisphosphonate, clinically widely used is zoledronate (Zometa, ZOL), a heterocyclic imidazole that inhibits osteoclastic bone resorption and possibly inhibits the tumor growth directly. Paclitaxel (taxol) is an antimicrotubule agent that promotes the assembly of microtubules from tubulin dimers and stabilizes microtubules by preventing depolymerization. It is one of the most potent agents against nonsmall cell lung cancer. Whether the combination of these two drugs can prevent bone metastasis and obtain the synergistic treatment result is not only notable, but also pressing.

The purpose of this study was to establish a human lung adenocarcinoma cell line with high bone metastatic potential 
in an immunodeficient mouse model and to evaluate the synergistic inhibitory activity of zoledronate and paclitaxel on bone metastasis in nude mice. The results of this study indicated that zoledronate enhanced the efficacy of paclitaxel synergistically, by reducing the incidence of bone metastasis from lung cancer and prolonging survival in a mouse model of non-small cell lung cancer with a high potential for metastasis to bone.

\section{Materials and methods}

Cell culture and in vivo selection. The SPC-A1 lung cancer cell line was established and well-characterized in our laboratory as previously reported (7). SPC-A1 cells $\left(10^{6}\right)$, grown in DMEM/10\% fetal calf serum, were injected by various routes into the left cardiac ventricle of NIH-BNX mice. Four weeks later, the mice were sacrificed and bonemetastasis-suspecious lesions were resected according to the bone scan. The general primary cell culture procedure was performed after the specimens were incised into small pieces. When the adherent cells were of sufficient quantity, they were trypsinized, collected in DMEM and re-injected into the left cardiac ventricle of mice. After 10 rounds of a similar in vivo selection, the human lung adenocarcinoma cells with high bone metastasis potential, SPC-A1-BM, was established.

Animal model and reagents. SPC-A1-BM cells, which were inoculated into the cardiac ventricle of $40 \mathrm{NIH}-\mathrm{BNX}$ mice, were purchased from the Shanghai Cancer Institute. Eight days after injection, they were randomized into 4 groups and were continuously treated with: a) normal saline (control), i.p.; b) zoledronate at $0.2 \mathrm{mg} / \mathrm{kg}$ mouse/day, twice per week, s.c.; c) paclitaxel, at $6.0 \mathrm{mg} / \mathrm{kg}$ mouse, first day every week, i.p.; d) the combination of paclitaxel and zoledronate, at the dose as above (8). Weights and performance status of the mice were evaluated biweekly. Mice were sacrificed if they were dying, otherwise they were sacrificed and dissected 8 weeks after implanting. The animal experimentation was in accordance with institutional guidelines.

Evaluation of bone metastasis using bone scan and X-rays. Bone metastasis in different treatment groups was evaluated with a bone scan and X-rays followed by a pathological examination. Before dissection, $148 \mathrm{MBq}$ 99Tc-MDP 3mCo 99mTc-MDP (Shanghai Xinke Company, Shanghai) was injected into the mice and a bone scan using SPECTE was performed to ascertain the overall distribution of the radiotracer. Bone metastasis was defined when hot spots were detected. X-ray (40 kv, 10 mA, 6 s, Philip Bncky Diagnost) was used and suspected bone destruction was noted.

Pathological evaluation and immunohistochemical staining. The mice were then sacrificed and liver, lungs as well as adrenal glands were surveyed for metastasis. Bilateral lungs were resected, pressured and counted for nodal number if there were any. The bone- metastasis- suspicious lesions and spine bones were immediately obtained and infiltrated into a $10 \%$ solution of formalin and delivered for the pathological procedure within $24 \mathrm{~h}$. A thick section $(4-\mu \mathrm{m})$ was cut for routing H\&E staining. For some cases, an antibody against cytokeratin was used for immunohistochemical staining and a positive finding was defined when the cytoplasm was stained.

With immunohistochemical staining, the expression of apoptosis and cell cycle related factors were analyzed after different treatments. In brief, tissue sections were deparaffinized with xylene for $5 \mathrm{~min}$, followed by two washes in absolute ethanol, 95 and $70 \%$ ethanol for $3 \mathrm{~min}$ each, then treated with $2 \% \mathrm{H}_{2} \mathrm{O}_{2}$ for $5 \mathrm{~min}$. The sections were blocked with diluted goat serum for $30 \mathrm{~min}$. Then, a regular ABC method was utilized to stain the section. Immunohistochemical staining includes p27 (dilution at 1:50, Cell Marque), Cyclin D1 (dilution at 1:300, Dako), Bcl-2 (dilution at 1:200, Dako), Bcl-xl (dilution at 1:200, Dako), Bax (dilution at 1:200, Dako). Sections were considered positive for P27, Cyclin D1, $\mathrm{Bax}, \mathrm{Bcl}-2$ and Bcl-xl when distinct nuclear staining was identified on cancerous cells in the bone. Each of the slides were examined independently by two specialists.

ELISA to measure NTX levels. Blood was collected and centrifuged at 2,000 x g for $10 \mathrm{~min}$ to obtain plasma, which was stored at $-20^{\circ} \mathrm{C}$ until further assay. ELISA kit to measure the n-telopeptide of type I collagen (NTX) levels was purchased from the Shanghai YuanXiang Medical Instrument Co., Ltd and the experiment was conducted strictly according to the manufacturer's instructions. The absorbances of the samples were measured at $492 \mathrm{~nm}$ on an Automated Microplate Reader (ELX 800, USA). The level of NTX was calculated according to the standard curve. All samples were run in a triplicate manner and the mean value was presented as a standard deviation.

Statistics. We used statistic software SPSS10.0. Survival curves were calculated according to the Kaplan-Meier method and compared by the log-rank test. Frequencies were compared using crosstabs and the Chi-squared test was used for qualitative values. Quantitive values were compared with ANOVA and the Q test was used between the two groups. $\mathrm{P}<0.05$ was considered to be statistically significant.

\section{Results}

The establishment of the SPC-A1-BM lung cancer line, which has high bone metastatic potential. The SPC-A1 cell line was established from a patient with lung adenocarcinoma and has been widely applied since (7). SPC-A1 was injected into the left cardiac ventricle, tail vain, or left lung of the NIH-BNX mice. The bone metastasis rate of parent SPC-A1 cells varied from 10.5 to $33 \%$, depending on the cell number and the method injected. A biopsy was performed on the suspected bone metastasis lesion and a primary cell culture from it was further conducted. After 10 rounds of repeated in vivo selection, the human lung adenocarcinoma cells with high bone metastatic potential, SPC-A1-BM was established, with a $100 \%$ bone metastasis rate when injected into the left cardiac ventricle of nude mice (Table I).

Inhibitory activity of zoledronate and paclitaxel on bone metastasis in nude mice. In terms of the bone metastasis shown by the bone scan, there was a significant difference when all 
Table I. Analysis of bone metastasis after tumor injection.

\begin{tabular}{|c|c|c|c|c|c|c|c|c|c|}
\hline \multirow[b]{2}{*}{ Generation } & \multicolumn{3}{|c|}{ Left cardiac ventricle } & \multicolumn{3}{|c|}{ Tail vein } & \multicolumn{3}{|c|}{ Left lung } \\
\hline & $\begin{array}{l}\text { Cells } \\
\left(\mathrm{x} 10^{4}\right)\end{array}$ & $\begin{array}{l}\text { Survival } \\
(\mathrm{t} / \mathrm{d})\end{array}$ & $\begin{array}{c}\text { Bone } \\
\text { metastasis }(\%)\end{array}$ & $\begin{array}{l}\text { Cells } \\
\left(\mathrm{x} 10^{4}\right)\end{array}$ & $\begin{array}{l}\text { Survival } \\
(\mathrm{t} / \mathrm{d})\end{array}$ & $\begin{array}{c}\text { Bone } \\
\text { metastasis }(\%)\end{array}$ & $\begin{array}{l}\text { Cells } \\
\left(\mathrm{x} 10^{4}\right)\end{array}$ & $\begin{array}{l}\text { Survival } \\
(\mathrm{t} / \mathrm{d})\end{array}$ & $\begin{array}{l}\text { Bone } \\
\text { metastasis }(\%)\end{array}$ \\
\hline Parent & 80 & 120 & $2 / 19(10.5)$ & & & & & & \\
\hline 1 & 100 & 40 & 2/8 (25) & 150 & 47 & 2/6 (33) & & & \\
\hline 2 & 100 & 21 & 4/6 (66.7) & 125 & 38 & $3 / 7(43)$ & & & \\
\hline 3 & 100 & 22 & $4 / 5(80)$ & 100 & 59 & $3 / 8(38)$ & 50 & 89 & 2/6 (33) \\
\hline 4 & 100 & 23 & $5 / 5(100)$ & 200 & 33 & 4/6 (67) & 100 & 35 & $2 / 4(50)$ \\
\hline 5 & 100 & 23 & 7/7 (100) & 175 & 49 & 4/8 (50) & 75 & 91 & $3 / 6(50)$ \\
\hline 6 & 100 & 20 & $6 / 6(100)$ & 100 & 61 & $3 / 7(43)$ & 50 & 78 & $5 / 9(56)$ \\
\hline 7 & 100 & 28 & 8/8 (100) & 150 & 56 & $5 / 9(52)$ & 50 & 75 & 4/7 (57) \\
\hline 8 & 100 & 29 & 8/8 (100) & 100 & 54 & 4/7 (57) & 70 & 67 & $6 / 10(60)$ \\
\hline 9 & 100 & 30 & 8/8 (100) & 150 & 59 & $6 / 10(60)$ & 100 & 64 & 4/6 (67) \\
\hline 10 & 100 & 30 & $8 / 8(100)$ & 100 & 55 & $5 / 7(71)$ & 50 & 65 & $5 / 9(56)$ \\
\hline
\end{tabular}

Tumor cells were injected into the left cardiac ventricle, tail vein and left lung, according to the schedule above and bone metastasis (bone mest) was measured 2 weeks after tumor injection. Animal survival was followed up.

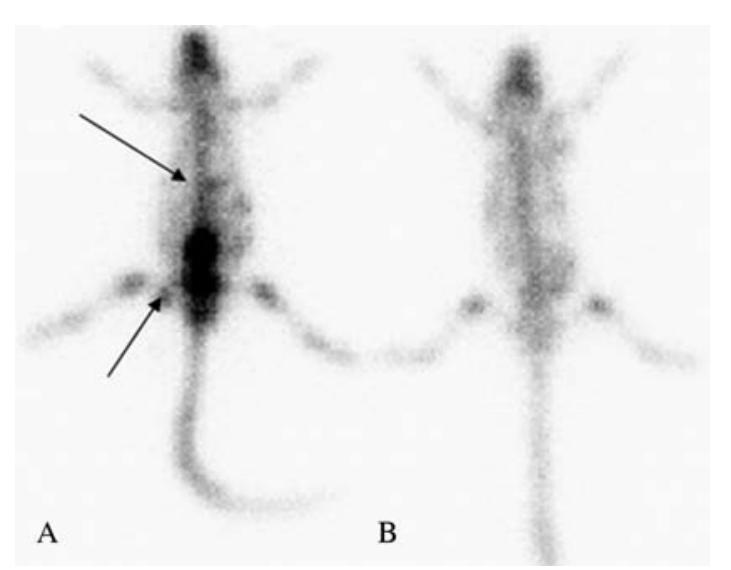

Figure 1. Bone metastasis shown by a nuclide scan. (A) Hot spots of bone metastasis shown in the nuclide scan. (B) Normal in the nuclide scan.

Table II. Bone metastasis shown by nuclide scan.

\begin{tabular}{lcc}
\hline & Negative \% (n) & Positive \% (n) \\
\hline Zometa & $60(6)$ & $40(4)$ \\
Taxol & $70(7)$ & $30(3)$ \\
Zometa + Taxol & $90(9)$ & $10(1)^{\mathrm{a}}$ \\
Control & $30(3)$ & $70(7)$ \\
\hline
\end{tabular}

$\mathrm{X}^{2}=8.000 ; \mathrm{P}=0.046$ and ${ }^{\text {a}}$ Fisher's $=0.020$.

four treatment groups were compared $(\mathrm{P}=0.048)$. This inhibitory activity was remarkable in the combined treatment group compared with the control group $(\mathrm{P}=0.020)$ and only one out of ten mice in the combined treatment group developed bone metastasis 8 weeks after incubation. Fig. 1 and Table II show bone metastasis illustrated by nuclide scan. Radiography
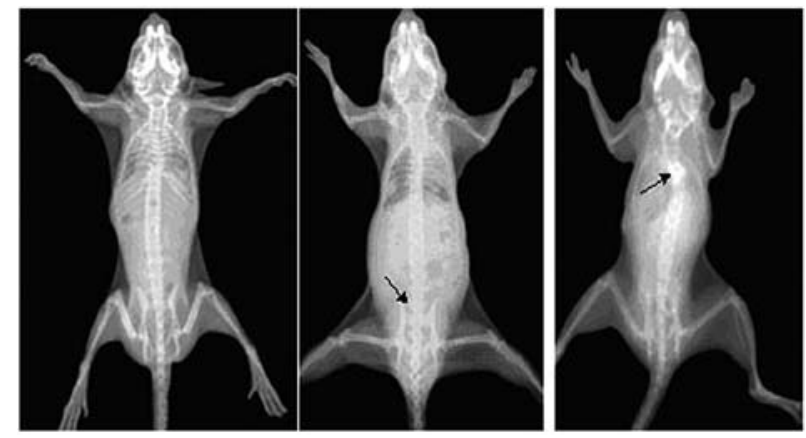

Figure 2. Bone metastasis shown by X-rays. Radiographically normal (left). Enlargement of sacral vertebrae shown by X-rays (middle). Destruction of sternum shown by $\mathrm{X}$-rays (right).

Table III. Bone metastasis shown by X-rays.

\begin{tabular}{lcc}
\hline & Negative \% (n) & Positive \% (n) \\
\hline Zometa & $80(8)$ & $20(2)$ \\
Taxol & $80(8)$ & $20(2)$ \\
Zometa + Taxol & $80(8)$ & $20(2)$ \\
Control & $30(3)$ & $70(7)$ \\
\hline
\end{tabular}

Combination treatment and control. $\mathrm{P}=0.036$.

was less sensitive in detecting bone metastasis in our study. Although no significant difference among four different groups was found for bone metastasis, the combined group still showed significantly less bone metastasis than the control group ( $\mathrm{P}=0.036$, Fig. 2 and Table III). Bulky metastatic disease in the bone of the control group was found under the microscopy, while only disseminated tumor cells in the treatment group were detected. In the zoledronate treatment 

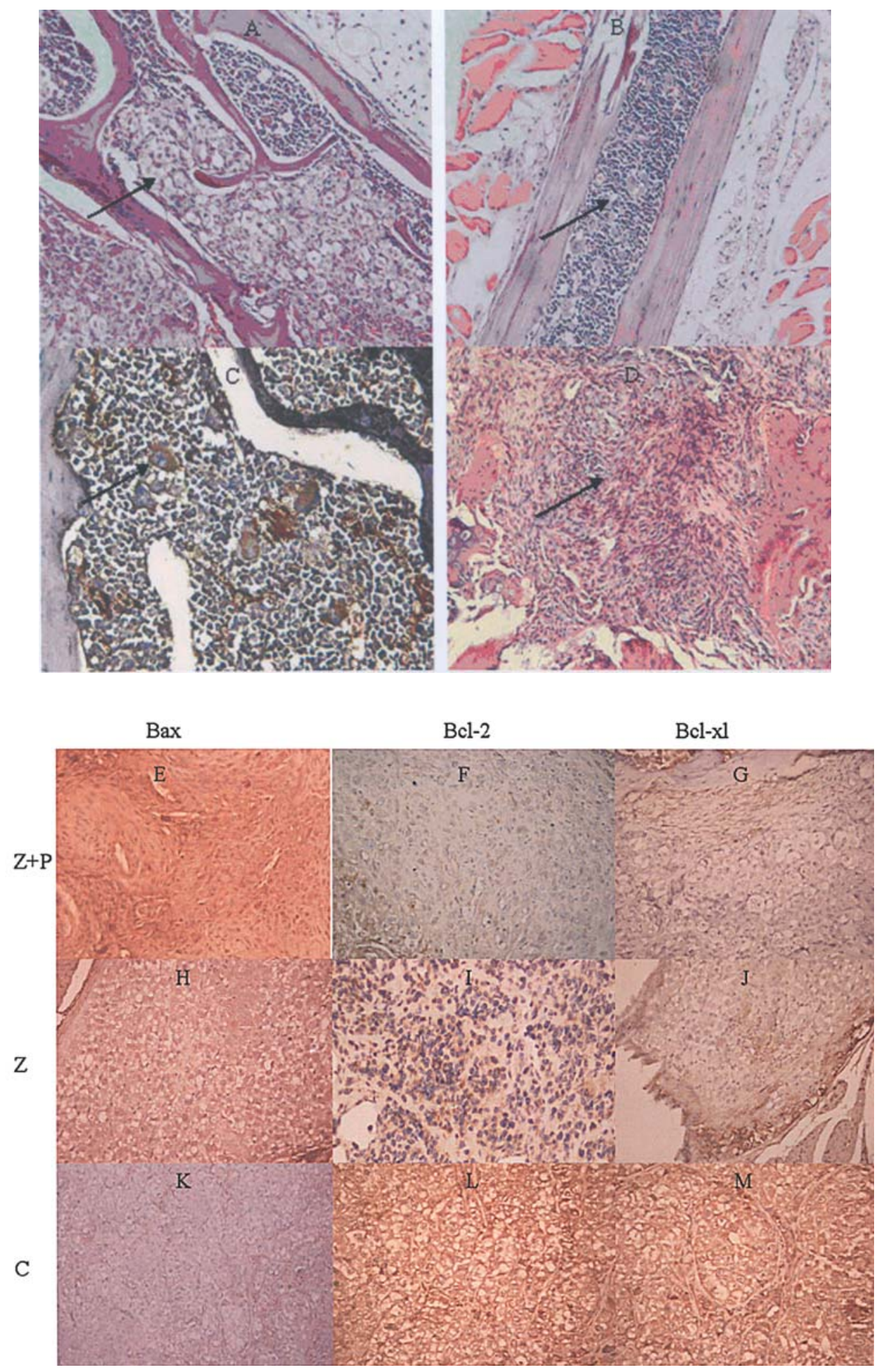

Figure 3. Bone metastasis detected by haematoxylin and eosin (H\&E) and immunohistochemical staining. (A) Bulky metastatic disease in the bone of the control group. (B) Cluster of disseminated tumor cells in the bone of the combined treatment group. (C) Metastatic tumor cells detected by anti-cytokeritin staining. (D) Repair of bone destruction by a granuloma-like component in the zoledronate treatment group. (E-G) The expression of Bax, Bcl-2 and Bcl-xl in paclitaxel (P) combined with the zoledronate (Z) treatment group shown by immunohistochemical staining. (H-J) The expression of Bax, Bcl-2 and Bcl-xl in the zoledronate (Z) treatment group. (K-M) The expression of Bax, Bcl-2 and Bcl-xl in the control C group. Compared with the control and the zoledronate treatment group, cancerous cells in the bone of the combined treatment group highly expressed Bax and expressed low levels of Bcl-2 as well as Bcl-xl. (x400 magnification).

group, repair of bone destruction by a granuloma-like component was found (Fig. 3A-D). There was a marginal difference $(\mathrm{P}=0.058)$ for bone metastasis confirmed by a histological analysis between the combination treatment and the control group. Compared with the control and zoledronate treatment group, cancerous cells in the bone of the combined treatment group highly expressed Bax and expressed low levels of Bcl-2 as well as Bcl-xl (Fig. 3E-M). The expression of cell cycle regulated factors, P27 and cyclin D1 did not change after a different intervention (data not shown). 


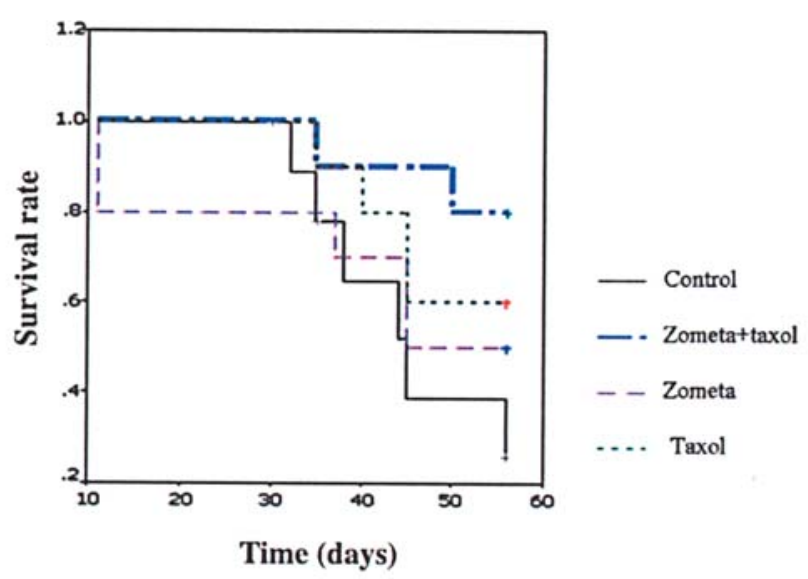

Figure 4. Survival rate in the different treatment groups. Survival curves were calculated according to the Kaplan-Meier method and compared by the log-rank test. The comparison of the four different groups showed no significant difference $(\mathrm{P}=0.1511)$, though the survival of the combined treatment group was significantly higher than that of the control group $(\mathrm{P}=0.022)$.
The median survival time of zoledronate alone, paclitaxel alone, the combination of paclitaxel and zoledronate and vehicle groups were 52, 45, 56 and 44 days, respectively. A comparison of the four different groups showed no significant difference $(\mathrm{P}=0.1511)$, though the survival of the combined treatment group was significantly higher than that of the control group ( $\mathrm{P}=0.022$, Fig. 4$)$.

Effect of the different treatments on the weight and other organ metastasis in SCID mice. Aside from the bone metastasis, mice injected with SPC-A1-BM cells developed lung, adrenal gland and lymph node metastasis, although they were less common (Fig. 5A). Lung metastasis was most frequent, occurring in 3, 3 and 6 mice in zoledronate alone, paclitaxel alone and vehicle groups. Notably, no mice were found to have lung metastasis in the combined treatment group.

After SPC-A1-BM was inoculated into the cardiac ventricle of the NIH-BNX mice, mice began to lose weight. Antitumor treatments seemed to prevent the mice losing weight. Eight weeks later, the mean weight of the mice in
A

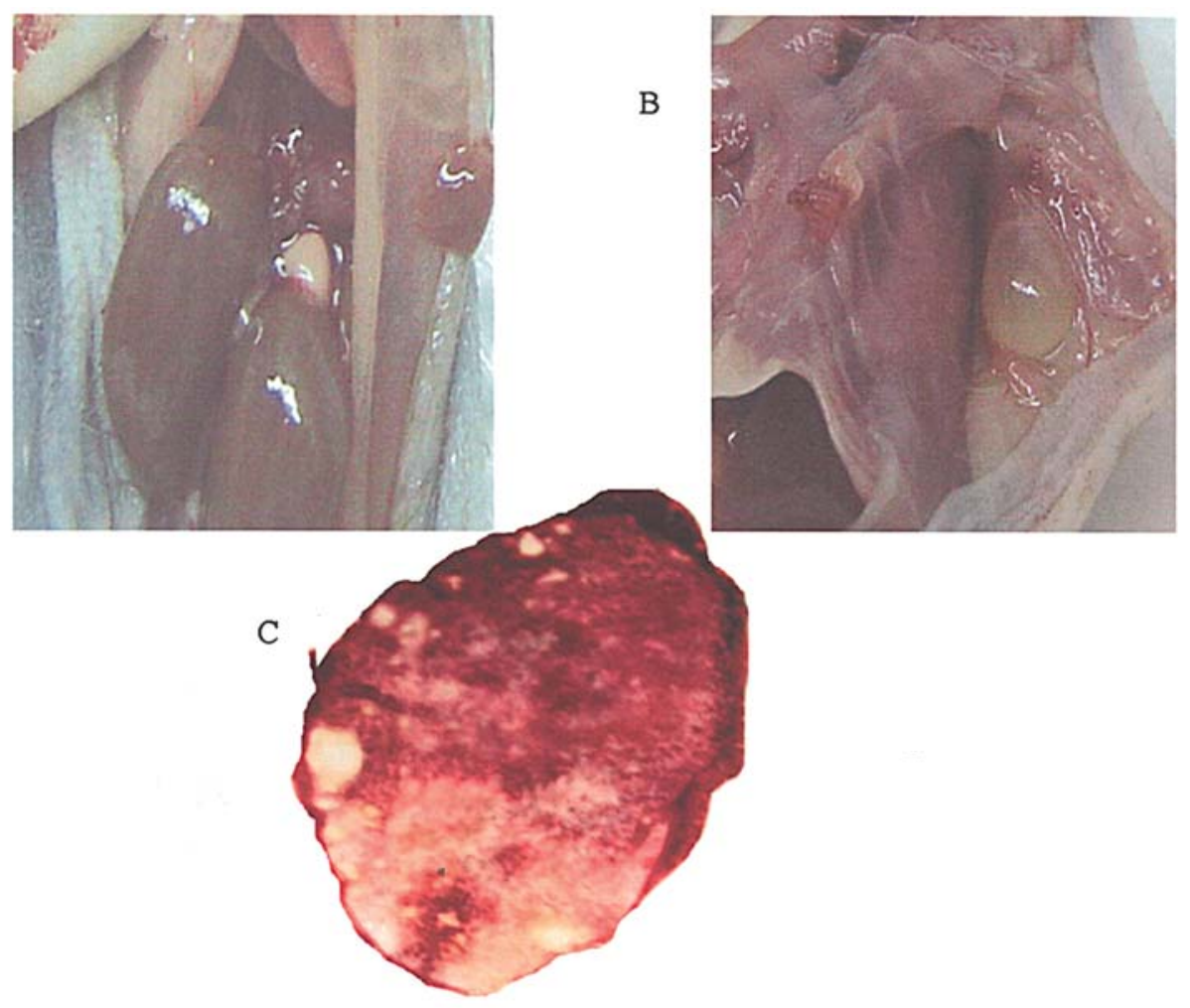

D

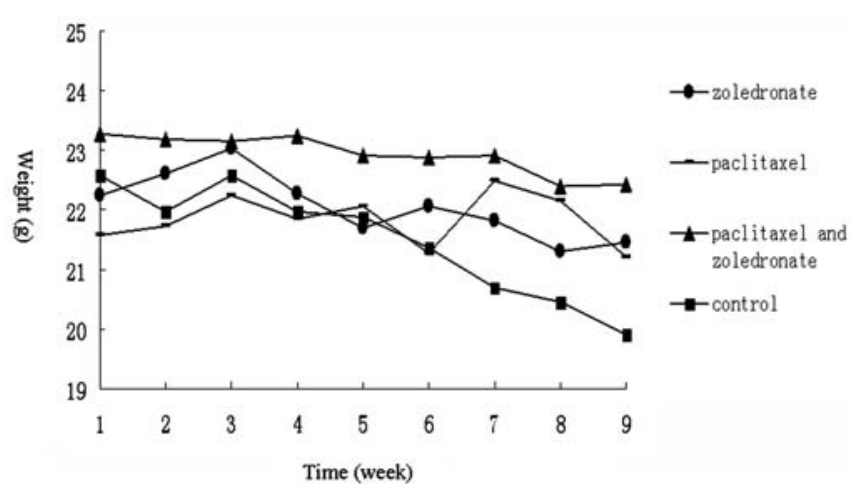

Figure 5. Other organ metastasis for high bone metastasis potential SPC-A1BM cells. (A) Malignant mass found on adrenal gland of the SCID mouse. (B) Enlargement of lymph nodes in the SCID mouse. (C) Wide-spread nodules in the lung of the SCID mouse. (D) A weight curve representing different treatments at different time points. 


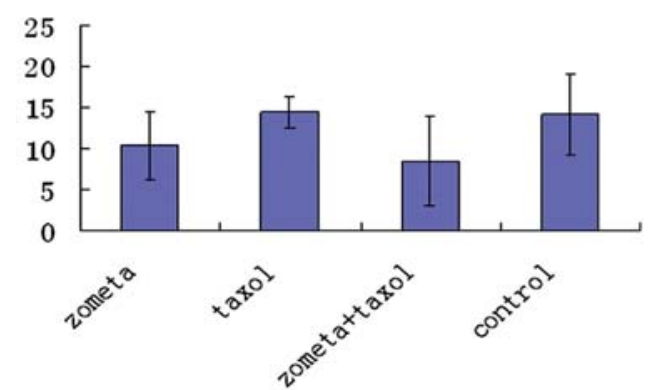

Figure 6. Reduction of the NTX level in the serum of nude mice by different treatments. The level of the NTX serum was measured by ELISA in a triplicate manner. The mean and standard deviation are presented.

zoledronate alone, paclitaxel alone, the combination of paclitaxel and zoledronate and vehicle groups were 21.44, 21.22, 22.43 and 19.92, respectively (paclitaxel combined with zoledronate and the vehicle group $\mathrm{P}=0.032$ ). Fig. $5 \mathrm{~B}$ shows the weight curve at different time points.

Reduction of NTX level in the serum of nude mice by different interferences. The measurement of biochemical markers of bone metabolism has become more common in patients with bone metastases. Decreases in marker levels have also been found to correlate with a response to systemic anticancer treatments (8). NTX is one of the bone turnover markers, which represents the activity of bone resorption. The mean serum level of bone turnover biomarker NTX in zoledronate alone, paclitaxel alone, the combination of paclitaxel and zoledronate and vehicle groups were 10.331, 14.419, 8.485 and $14.193 \mathrm{nM}$, respectively (Fig. 6). The NTX level was significantly reduced by the treatment of zoledronate combined with paclitaxel compared with the control group $(\mathrm{P}=0.022)$.

\section{Discussion}

Currently available palliative therapies for patients with malignant bone disease include radiation, chemotherapy, hormone therapy, orthopedic surgery and i.v. bisphosphonates. Bisphosphonate therapy has emerged as an important component of the overall management strategy for malignant bone disease (6). There are still a couple of questions, which need to be addressed regarding the application of bisphosphonate. In this study, we established a nude mouse model which has high risk of bone metastasis. We hope to solve these questions using this animal model and give some hints for future daily practice.

The first question to be resolved is when to start the bisphosphonate for the patients with cancer. The panel suggestion from the American Society of Clinical Oncology is that bisphosphonate therapy should be initiated at the first radiographic evidence of bone destruction or an abnormal bone scan with localized pain. There is increasing evidence that bisphosphonate may be beneficial even earlier in the course of therapy, before overt bone metastases occur in breast cancer and prostate cancer patients (9-11). As far as we know, it is still unknown whether bisphosphonate can prevent lung cancer cells spreading to the bone in a clinical setting.
In this current study, either zoledronate alone, or combined with paclitaxel significantly reduced bone metastasis. Our results indicated that a prophylactic application of zoledronate is useful before bone metastases develops in the animal model. An ongoing prospective international clinical trial, CZOL446G2-419, will elucidate whether bisphosphonate can prevent the incidence of bone metastasis in stage IIIA or IIIB lung cancer patients who have completed primary treatment.

There have been some reports on whether zoledronic acid can be enhanced by cytotoxic regiments. Ozturk et al reported that cisplatin cytotoxicity, though not gemcitabine was enhanced with zoledronate in the A549 lung cancer cell line (12). Whereas, Knight et al reported a simultaneous addition of zoledronate to cisplatin or paclitaxel showed no major increase in cytotoxicity in cancer cell lines including breast, lung and ovarian (13). It is reasonable that the results were heterogeneous since all of the above studies were conducted on the cell line level. Our experiment is the first to study the synergistic effects of chemotherapy and zoledronic acid in an NSCLC animal model. Our results showed that compared with paclitaxel alone, zoledronate combined with paclitaxel tended to reduce bone and lung metastasis and prolongs the median survival time, although the difference was not significant. Paclitaxel and zoledronate at the dose used not only works well with mice, but also reduced the symptoms of cancerous cachexia. In a clinical setting, zoledronate proved to reduce skeletal-related events in patients of lung cancer with bone metastases, though time to progression in zoledronate plus the chemotherapy group was identical to that in the chemotherapy group alone (14). More pre-clinical experiments and prospective clinical trials are needed to elucidate whether zoledronate plus chemotherapy is superior to chemotherapy alone in advanced NSCLC.

In our study, compared with the control and zoledronate treatment groups, cancerous cells in the bone overexpressed Bax and expressed low levels of Bcl-2 and Bcl-xl after combined treatment. This indicated paclitaxel and zoledronate possibly exerted synergistic inhibitory activity through inducing programmed cell death. The underlying mechanism of zoledronate activity was investigated previously $(15,16)$. Zoledronate induced cell cycle arrest of the osteosarcoma POS-1 cell line by inhibiting it in the S phase (17). Paclitaxel can inhibit the cell cycle by $\mathrm{M}$ phase arrest. Therefore they can theorically exert an antitumor effect by different checkpoint arrests. Fromigué et al reported that the combination of ZOL with paclitaxel resulted in synergistic apoptotic effects in breast cancer cell lines (18).

Bone turnover markers refer to biochemical moleculars reflecting bone metabolism. An assessment of these markers provides specific insight into rates of bone formation and resorption (19-22). The results of a clinical study suggest that elevations in bone resorption markers are associated with the severity of bone pain and the extent of bone metastases (23-26). Our results showed that the NTX level was reduced by zoledronate compared with the control group in the animal model. The activity was remarkable in the combined group. Recently, it was shown that patients with bone metastases from lung cancer who had high NTX or BALP levels in the study had a higher risk for skeletal-related events and 
decreased survival compared with patients with low NTX or BALP $(23,27)$. The treatment of bisphosphonate did decrease the level of NTX. NTX could potentially be the predictive maker which monitors the treatment efficacy.

\section{Acknowledgements}

This study was supported, in part, by the Shanghai Lung Cancer Center Grant, Shanghai Municipal Natural Science Fund 072R (to S.L.), Shanghai Cancer Hospital Grants YX 200602, KY176 and the Chinese post-doc fellowship 20070410733 (to J.Z.). Supported by Shanghai Leading Academic Discipline Project (B115).

\section{References}

1. van der Pluijm G, Sijmons B, Vloedgraven $\mathrm{H}$, et al: Monitoring metastatic behavior of human tumor cells in mice with species specific polymerase chain reaction: elevated expression of angiogenesis and bone resorption stimulators by breast cancer in bone metastases. Bone Miner Res 16: 1077-1091, 2001.

2. Hauschka PV, Mavrakos AE, Iafrati MD, et al: Growth factors in bone matrix: isolation of multiple types by affinity chromatography on heparin-sepharose. J Biol Chem 261: 12665-12674, 1986.

3. Coleman RE: Skeletal complications of malignancy. Cancer 80: 1588-1594, 1997

4. Bloomfield DJ: Should bisphosphonates be part of the standard therapy of patients with multiple myeloma or bone metastases from other cancers? An evidence-based review. J Clin Oncol 16: 1218-1225, 1998

5. Roodman GD: Mechanisms of bone metastasis. N Engl J Med 350: 1655-1664, 2004

6. Rogers MJ, Watts DJ and Russel RG: Overview of bisphosphonates. Cancer 80: 1652-1660, 1997.

7. Chen Z, Wang X, Gao L, et al: Regulation of MUC5AC mucin secretion by depletion of AQP5 in SPC-A1 cells. Biochem Biophys Res Commun 342: 775-781, 2006.

8. Zhou Z, Guan H, Duan X, et al: Zoledronic acid inhibits primary bone tumor growth in Ewing sarcoma. Cancer 104: 1713-1720, 2005.

9. James R: Berenson recommendations for zoledronic acid treatment of patients with bone metastases. Oncologist 10: 52-62, 2005.

10. Smith MR, Eastham J, Gleason DM, et al: Randomized controlled trial of zoledronic acid to prevent bone loss in men receiving androgen deprivation therapy for nonmetastatic prostate cancer. J Urol 169: 2008-2012, 2003.

11. Diel IJ, Solomayer EF, Costa SD, et al: Reduction in new metastases in breast cancer with adjuvant clodronate treatment. N Engl J Med 339: 357-363, 1998.
12 Ozturk OH, Bozcuk H, Burgucu D, et al: Cisplatin cytotoxicity is enhanced with zoledronic acid in A549 lung cancer cell line: preliminary results of an in vitro study. Cell Biol Int 31: 1069-1071, 2007.

13. Knight LA, Conroy M, Fernando A, et al: Pilot studies of the effect of zoledronic acid (Zometa) on tumor-derived cells ex vivo in the ATP-based tumor chemosensitivity assay. Anticancer Drugs 16: 969-976, 2005.

14. Rosen LS, Gordon D, Tchekmedyian S, et al: Zoledronic acid versus placebo in the treatment of skeletal metastases in patients with lung cancer and other solid tumors: a phase III, double-blind, randomized trial-the zoledronic acid lung cancer and other solid tumors study group. J Clin Oncol 21: 3150-3157, 2003.

15. Kunzmann V, Bauer E, Feurle J, et al: Stimulation of $\gamma \delta \mathrm{T}$ cells by aminobisphosphonates and induction of antiplasma cell activity in multiple myeloma. Blood 96: 384-392, 2000.

16. Dieli F, Gebbia N, Poccia F, et al: Induction of gammadelta T-lymphocyte effector functions by bisphosphonate zoledronic acid in cancer patients in vivo. Blood 102: 2310-2311, 2003.

17. Ory B, Heymann MF, Kamijo A, et al: Zoledronic acid suppresses lung metastases and prolongs overall survival of osteosarcoma-bearing mice. Cancer 104: 2522-2529, 2005.

18. Fromigué O, Lagneaux L and Body JJ: Bisphosphonates induce breast cancer cell death in vitro. J Bone Miner Res 15: 2211-2221, 2000 .

19. Costa L, Demers LM, Gouveia-Oliveira A, et al: Prospective evaluation of the peptide-bound collagen type I cross-links Ntelopeptide and C-telopeptide in predicting bone metastases status. J Clin Oncol 20: 850-856, 2002.

20. Aruga A, Koizumi M and Hotta R: Usefulness of bone metabolic markers in the diagnosis and follow-up of bone metastasis from lung cancer. Br J Cancer 76: 760-764, 1997.

21. Horiguchi T, Tachikawa S, Kondo R, et al: Usefulness of serum carboxy-terminal telopeptide of type I collagen (ICTP) as a marker of bone metastasis from lung cancer. Jpn J Clin Oncol 30: $174-179,2000$.

22. Izumi M, Nakanishi Y, Takayama K, et al: Diagnostic value of bone-turnover metabolites in the diagnosis of bone metastases in patients with lung carcinoma. Cancer 91: 1487-1493, 2001.

23. Coleman RE, Major P, Lipton A, et al: Predictive value of bone resorption and formation markers in cancer patients with bone metastases receiving the bisphosphonate zoledronic acid. J Clin Oncol 23: 4821-4822, 2005.

24. Santini D, Vincenzi B, Hannon RA, et al: Changes in bone resorption and vascular endothelial growth factor after a single zoledronic acid infusion in cancer patients with bone metastases from solid tumours. Oncol Rep 15: 1351-1357, 2006.

25. Coleman RE: Bone markers in metastatic bone disease. Cancer Treat Rev 32: 27-28, 2006.

26. Papotti M, Kalebic T, Volante M, et al: Bone sialoprotein is predictive of bone metastases in resectable non-small-cell lung cancer: a retrospective case-control study. J Clin Oncol 24: 4818-4824, 2006

27. Roodman GD: High bone turnover markers predict poor outcome in patients with bone metastasis. J Clin Oncol 23: 4925-4935, 2005. 